\title{
BEAMS FOR GAMMASPHERE AT THE LBNL 88-INCH CYCLOTRON
}

\author{
D.J. Clark, D. Collins, R. Dwinell, A. Guy, S. Lundgren, C.M. Lyneis, M.A.McMahan, D. \\ Syversrud and Z. Q. Xie, Lawrence Berkeley National Laboratory
}

\section{Abstract}

The new transport system from the 88-Inch Cyclotron to the Gammasphere facility is described. Included are discussions of the ECR sources, operation, beam diagnostics, mechanical construction, vacuum system and beam optics.

\section{INTRODUCTION}

The 88-Inch Cyclotron at Lawrence Berkeley National Laboratory is the present home of Gammasphere, a powerful gamma ray array for studying the structure of nuclei. Gammasphere has been operating as a national facility since April of 1993, running 104 major experiments and over 12,000 hours since then. The majority of Gammasphere experiments run to date have used compound nucleus reactions at energies just above the Coulomb barrier $(\approx 5 \mathrm{MeV} / \mathrm{u})$ to make specific nuclei of interest for nuclear structure studies, especially nuclei that have been found or predicted to exhibit very large deformations at high spins, called superdeformed (with an axis ratio of 2:1) or hyperdeformed (with an axis ratio of $3: 1$ ). In order to make these nuclei, beams of medium mass are generally used, and often rare isotopes are required. Modest intensities are needed, on the order of $\leq$ 5 pnA at the target. Other Gammasphere experiments have used transfer reactions to make nuclei far from stability and study their structure. Here much heavier beams are needed at higher energies. To date, the heaviest beam run for these experiments has been ${ }^{162} \mathrm{Dy}$.
Gammasphere running has occupied $52 \%$ of the scheduled Cyclotron time.

In order to meet the needs of these experiments, a new beamline was constructed with improved vacuum, beam diagnostics, and optics systems, shown in Fig. 1.

\section{OPERATION}

The ECR [1] and AECR-U [2] sources supply heavy ion beams to the Cyclotron. The ECR source started regular operation in 1985. It is a 2-stage source with the main stage operating at $6.4 \mathrm{GHz}$. It can use either of two types of ovens, with temperatures up to 700 deg. C and $2000 \mathrm{deg}$. C to provide solid material feed radially into the plasma. The AECR-U source has a single stage operated originally at $14 \mathrm{GHz}$. It has been upgraded by increasing the magnetic field, using multiple frequencies of 14 and $10 \mathrm{GHz}$ and using an aluminum vacuum chamber. With the AECR-U the Cyclotron can now supply beams to Gammasphere up to mass 200 at 5 $\mathrm{MeV} / \mathrm{u}$. Both high and low temperature ovens are available for the source, with 2 radial insertion positions. The source-cyclotron system is efficient enough to run rare isotopes such as ${ }^{54} \mathrm{Cr}$ and ${ }^{76} \mathrm{Ge}$ from natural feed material. ${ }^{36} \mathrm{~S}$ is enriched to $2.5 \%$ and ${ }^{48} \mathrm{Ca}$ to $39 \%$. The overall beam delivery system to Gammasphere has had a $98 \%$ uptime.

During beam development using a sample 200 $\mathrm{MeV} \mathrm{Ar}^{9+}$ beam, the optics were refined, and a Microsoft Excel GENTUNE predictive spreadsheet for

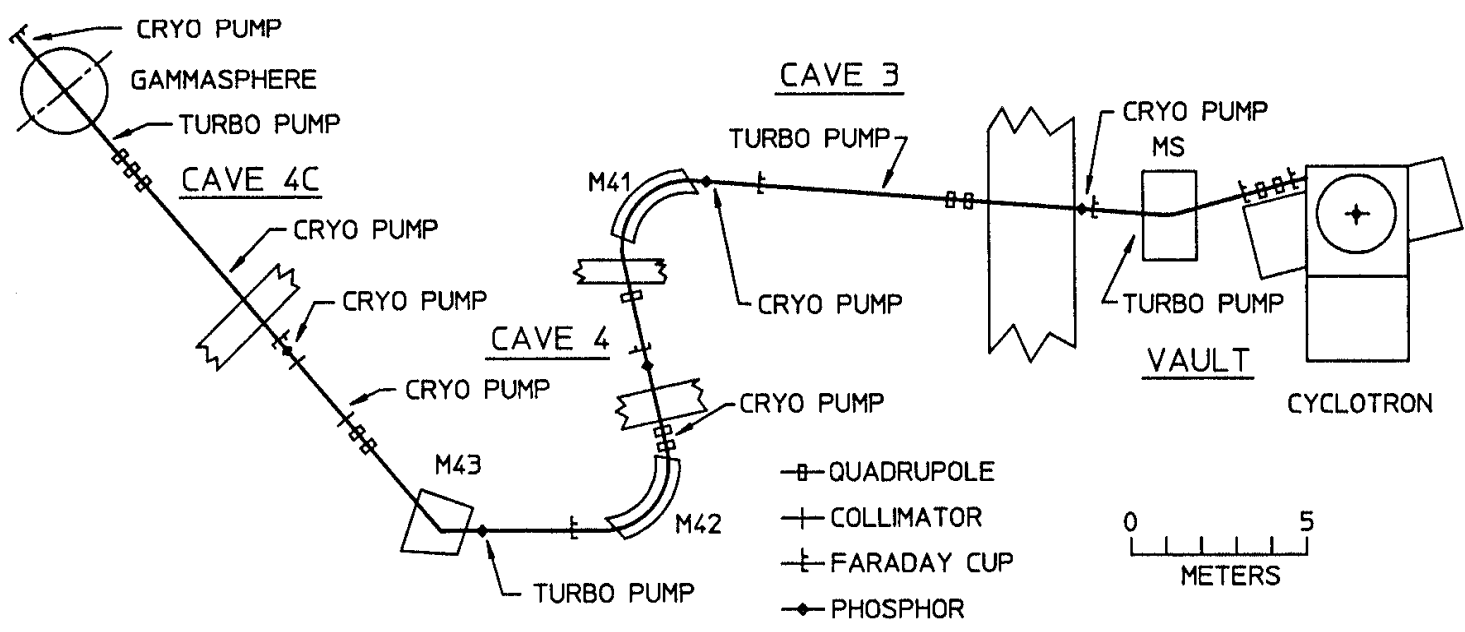

Figure: 1 Mechanical layout of beamline. 


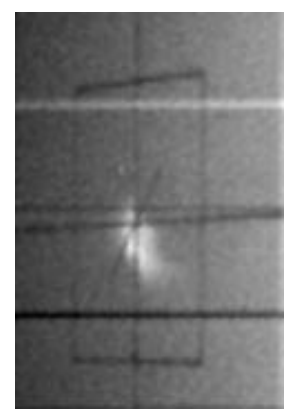

Figure: 2 Beam spot on phosphor at focus in Cave 4.

quadrupoles and bending magnets was created for all reasonable combinations of ion species and energy. This supplanted the older QuickBasic MASTER program.

Five $\mathrm{ZnS}$ phosphors provide first level optic diagnostics, with beam levels typically around 100 epA. Six magnet-suppressed Faraday cups are available for checking transmission, and 11 combinations of adjustable slits or plungeable collimators can be used to define beam spot size and to reduce halo on target.

Once the Cyclotron tune is completed and the GENTUNE values are loaded, the remaining optics tuning is very quick, typically around five minutes from Cyclotron exit until the beam is in Gammasphere. Focusing is not usually done on the phosphors; beam spot shape and position are compared on a go/nogo basis against benchmarks. Optimizing the tune on target is done typically by reducing the beam on a collimator at the target, relative to the beam on a Faraday cup at the end of the line. Fulfilling even the most complex requirements typically take less than 20 minutes. The transport from the cyclotron along the $50 \mathrm{~m}$ beamline to the target reaches 50\% transmission with careful tuning. The predicted beam size on the target is $3 \mathrm{~mm}$ diameter. This has been verified experimentally. The beam size can be increased by defocusing the beam with the triplet just before Gammasphere. The beam spot on a phosphor at the last focus before Gammasphere in Cave 4 is shown in Fig. 2. The rectangular box is $4 \mathrm{~cm} \mathrm{x} 4 \mathrm{~cm}$. The phosphor is mounted at $45 \mathrm{deg}$. to the beam direction. Normally, a $.75 \mathrm{~cm}$ collimator is plunged here to define the spot size, transmitting most of the beam while removing most of the halo.

The beam pulse width can be reduced to 1-2 ns FWHM by using cyclotron internal beam collimators and a Fast Faraday Cup described in the next section.

\section{BEAM DIAGNOSTICS}

Phosphors, Faraday cups, fixed diameter beam collimators and guillotines (beam stops) can be mounted to standardized plunging drives. They are in-house designed units which are controlled from the control room. Color coding of the external support bracket helps to identify which type of internal device is in use. Adjustable slits are electric motor driven with linear potentiometer position sensing and are controlled from the control room.
A fast response Faraday cup is normally placed in the downstream end of Cave 4. It was designed and constructed by cyclotron staff. It uses a $50 \mathrm{ohm}$ line at the beam stop, tapering down to a $50 \mathrm{ohm}$ cable at the back of the cup, which carries the signal through a vacuum seal. The signal then goes through a fast amplifier (HP 8447F), to a low loss Heliax cable to bring the signal to a sampling oscilloscope (Tektronix TDS $820)$ in the Control Room. In the control room the various cyclotron parameters can be tuned for narrow phase width when desired, while watching the beam pulse on the scope. Two retractable slits inside the cyclotron at a beam radius of 5-15 inches are useful to define the beam phase width. Each slit has two blades with separate drives for gap width and radius position. Using these slits the phase could be reduced to 1-2 nanoseconds FWHM for Gammasphere type beams of 5-6 MeV/u, where cyclotron frequencies are $15-16 \mathrm{MHz}$.

In order to provide a test of the beam pulse width independent of the fast Faraday cup, and to be able to measure lower-intensity beams, a small, plungeable scintillator/photo-tube assembly was installed just prior to the fast Faraday cup in the Cave $4 \mathrm{C}$ beam line. This scintillator measured the timing of the beam relative to the RF. Since it detects individual beam particles it normally needs a beam attenuator upstream. The width of the beam pulse was comparable to that measured by the fast Faraday cup.

A $\mathrm{BaF}$ gamma ray detector has also been used outside the beam pipe at the same location to measure beam pulse shape by detecting gammas from a beam stop. It gave similar results to the above methods.

\section{MECHANICAL SYSTEMS}

\subsection{Earthquake Safety}

Quadrupole magnets which were added to the beam line were first aligned, then bolted to the floor for an earthquake safe installation. Existing quadrupole installations remain counter weighted to prevent tipping during an earthquake. Extensive reinforcement of the Gammasphere cave shielding was done for earthquake protection.

\subsection{Vacuum System}

The vacuum system uses stainless steel beam pipe, aluminum beam boxes and turbo and cryopumps. The stainless steel beam pipe sections making up the $50 \mathrm{~m}$ long beamline were electropolished and UHV cleaned for improved vacuum. The aluminum vacuum pumping stations and diagnostic boxes were UHV cleaned for high vacuum service. Fluorocarbon rubber is used for both static and dynamic seals to reduce outgassing. Beam line isolation valves are interlocked on upstream and down stream pressures. They have position indication and control both locally and in the Control Room.

Roughing the beam line is accomplished by a system of locally controlled vacuum pumping stations utilizing turbomolecular pumps, with pumping speeds of $300-5001 / \mathrm{s}$ for nitrogen, backed with rotary vane pumps. 
For the final vacuum the turbopumps continue to pump the beamline and a system of cryopumps is added, each with a pumping speed of $1500 \mathrm{l} / \mathrm{s}$ for nitrogen. Each pump is locally controlled and is connected to the beamline through an interlocked gate valve for isolation during servicing. The cryopumps are regenerated by a dry pump or $\mathrm{LN}_{2}$ trapped rotary vane pump. Pumpdown time from atmosphere to operating pressure is 15 minutes. Bayard-Alpert ionization gauges positioned midway between pumping stations give indication of the beamline pressures of $1 \times 10^{-6}$ to $1 \times 10^{-7}$ torr after a day of pumping.

Beamlines are vented to atmospheric pressure using dry nitrogen which is piped from the main $\mathrm{LN}_{2}$ tank vaporizer/economizer system, providing 7.9 1/s (1000 cu. ft./hr.) of dry nitrogen at $3.1 \times 10^{5}$ pascals (45 psi) throughout the experimental areas. Venting to dry nitrogen has improved the pumpdown speed and the final pressure.

\section{BEAM OPTICS}

The new optics was designed to meet the following requirements of beam on target:

1. Beam emittance on target is minimum: no energy spread contribution.

2. Beam spot size on target is as small as $3 \mathrm{~mm}$ diameter for some experiments.

3. Maximum beam divergence is adjustable.

4. Transmission from ion source to target should be maximum, to make possible runs with low source intensity (rare isotopes or high mass).

5. Beam collimators producing background radiation should be kept away from the target, preferably in an upstream cave.

The resulting solution is shown in Fig. 3. The half beam envelopes are shown for the " $\mathrm{x}$ " or horizontal direction in the top half of the plot, and for the "y" or vertical direction in the bottom half of the plot. The top and bottom boundaries of the plot space at $5 \mathrm{~cm}$ represent the beam pipe radius for the standard 4 inch diameter pipe and the quadrupoles. $\operatorname{Eta}(\mathrm{x})$ is the dispersion, or $\Delta \mathrm{p} / \mathrm{p}$ ray.

To meet the condition of minimum emittance at the target, the $\Delta \mathrm{p}$ ray should cross the axis at the target and be travelling along the axis. The solution uses an additional quad singlet, and a doublet, between M41 and M42. These quads keep the $\Delta \mathrm{p}$ ray to small amplitude and give the desired trajectory along the axis after the last dispersive element, M43. A central ray with a momentum displacement $\Delta \mathrm{p} / \mathrm{p}$ of $.075 \%$ is shown diverging from the axis, slightly in MS and more in M41 and M42. This $\Delta \mathrm{p}$ ray represents the beam energy spread, which adds to the horizontal size of the beam. A quad triplet is used in Cave 4C to give good control over the beam spot shape on target. The triplet is placed close to the target to get the demagnification for a small spot size. For Gammasphere optics, this brought the total number of elements to 12 quadrupoles, four large bending magnets, and ten small steering magnets.
The requirement of keeping the last collimators away from the target is met by having them behind the Cave 4C wall in the upstream cave, Cave 4. They are plungeable from the Control Room. They consist of 3 plates with different hole sizes for forming an object for the quad triplet in Cave 4C. Selection of the hole size gives control over the beam spot size on target. Another set of collimators is further upstream, near the previous quad. These are 4 independent jaws, controllable from the Control Room, which control divergence of the beam on target by controlling beam size at the position in the previous cave where the beam is large.

The design of the beam line has satisfied all the requirements, and this solution is now the one routinely used for Gammasphere. This beam optics was designed using the LATTICE code, a user friendly version of TRANSPORT written by J. Staples of LBNL.

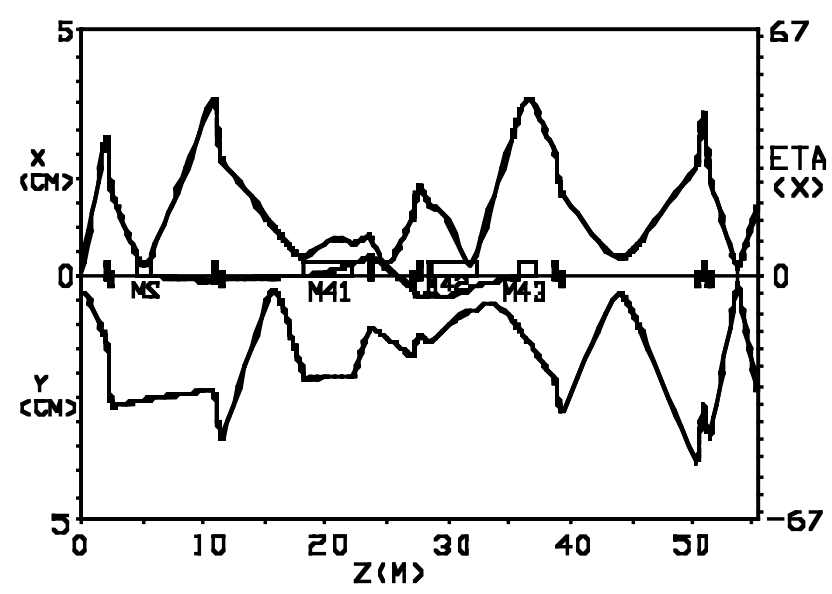

Figure: 3 Beam optics layout.

\section{ACKNOWLEDGEMENTS}

The authors wish to thank the Cyclotron engineering groups, shops, and crew for the successful completion of this project. G. Wozniak and M. Maier made important contributions to the beam pulse width measurement. This work was supported by the Director, Office of Energy Research, Division of Nuclear Physics of the Office of High Energy and Nuclear Physics of the U.S. Department of Energy under Contract DE- ACO3$76 \mathrm{SF} 00098$.

\section{REFERENCES}

[1] C.M. Lyneis, Proceedings of the International Conf. on ECR Ion Sources and their Applications, East Lansing, NSCL Report \#MSUCP-47, 1987, p. 42.

[2] Z. Q. Xie and C. M. Lyneis, Proceedings of the 13th International Workshop on ECR Ion Sources, College Station, Texas, Feb 1997, to be published. 